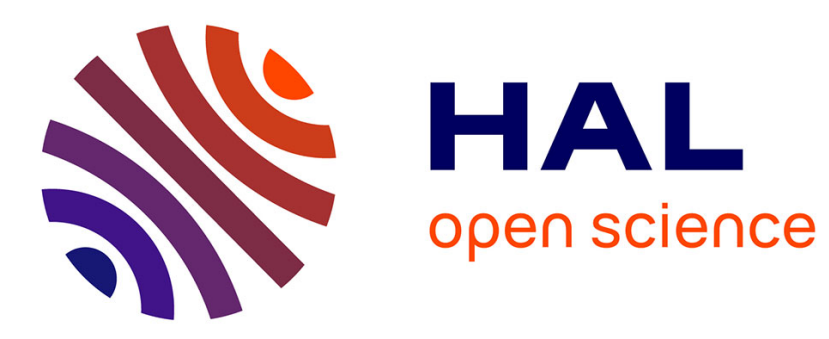

\title{
Energy distribution and cooling of a single atom in an optical tweezer
}

\author{
C Tuchendler, Andrew Matheson Lance, Antoine Browaeys, Yvan R. P. \\ Sortais, Philippe Grangier
}

\section{- To cite this version:}

C Tuchendler, Andrew Matheson Lance, Antoine Browaeys, Yvan R. P. Sortais, Philippe Grangier. Energy distribution and cooling of a single atom in an optical tweezer. Physical Review A : Atomic, molecular, and optical physics [1990-2015], 2008, 78, pp.033425 / arXiv: 0805.3510. 10.1103/PhysRevA.78.033425 . hal-00509055

\section{HAL Id: hal-00509055 https://hal.science/hal-00509055}

Submitted on 14 Jan 2015

HAL is a multi-disciplinary open access archive for the deposit and dissemination of scientific research documents, whether they are published or not. The documents may come from teaching and research institutions in France or abroad, or from public or private research centers.
L'archive ouverte pluridisciplinaire HAL, est destinée au dépôt et à la diffusion de documents scientifiques de niveau recherche, publiés ou non, émanant des établissements d'enseignement et de recherche français ou étrangers, des laboratoires publics ou privés. 


\title{
Energy distribution and cooling of a single atom in an optical tweezer
}

\author{
C. Tuchendler, A. M. Lance, * A. Browaeys, Y. R. P. Sortais, and P. Grangier \\ Laboratoire Charles Fabry de l'Institut d'Optique, CNRS, Université Paris-Sud, Campus Polytechnique, RD 128, \\ 91127 Palaiseau Cedex, France
}

(Received 22 May 2008; published 23 September 2008)

\begin{abstract}
We investigate experimentally the energy distribution of a single ${ }^{87} \mathrm{Rb}$ atom trapped in a strongly focused dipole trap under various cooling regimes. Using two different methods to measure the mean energy of the atom, we show that the energy distribution of the radiatively cooled atom is close to thermal. We then demonstrate how to reduce the energy of the single atom, first by adiabatic cooling, and then by truncating the Boltzmann distribution of the single atom. This provides a nondeterministic way to prepare atoms at low micro-K temperatures, close to the ground state of the trapping potential.
\end{abstract}

DOI: 10.1103/PhysRevA.78.033425

PACS number(s): 37.10.Jk, 42.50.Ct, 03.67.-a

\section{INTRODUCTION}

In the last thirty years, the manipulation of single quantum objects has been the subject of considerable attention. After the pioneering experiments of Dehmelt with a single electron in a Penning trap [1], a single ion was trapped in a Paul trap [2], which led to the observation of quantum jumps $[3,4]$. Several techniques were then developed to cool the single trapped particle, such as optical sideband cooling, which was experimentally demonstrated in 1989 [5]. More recently, the interest in the manipulation of single quantum objects has grown with the recognition that they can carry quantum information, and that they therefore can be a resource for quantum information processing [6]. In this context, single trapped laser cooled ions have accumulated an impressive amount of results in recent years (see, e.g., Ref. [7]).

The trapping of single neutral atoms, on the other hand, is more recent, owing in part to their weaker interaction with the electromagnetic field. So far, the trapping of neutral atoms has been realized in tight magneto-optical traps $[8,9]$ or optical dipole traps [10-13]. As the trapping potentials are typically less than a milli-K deep, laser cooling is the usual starting point of the manipulation of single atoms. As is the case for ions, it is tempting to further reduce the energy of the trapped atom, to ultimately reach the ground vibrational state of the trapping potential. In the framework of quantum computing, for example, the entanglement of two atoms via controlled collisions usually requires ground state cooling (see, e.g., Ref. [14]).

The knowledge and control of the energy of a single atom, trapped in a submicron optical dipole trap, is important for many purposes. For instance, in a recent experiment [15], we have used two single rubidium atoms, trapped in two optical tweezers separated by a few microns, as single-photon sources. When the two photons (each photon coming from one atom) are superimposed on a beamsplitter, we observe a "coalescence" effect: the two (indistinguishable) photons copropagate from the same output of the beamsplitter, due to a two-photon interference effect. We have shown that the ulti-

\footnotetext{
*andrew.lance@institutoptique.fr
}

mate visibility of this effect is limited by the spread in frequencies of the emitted photons, which in turn is due to the spread in energy of the emitting trapped atoms. In another experiment [16], using Raman transitions we have prepared a single atom in a superposition of two internal states of the hyperfine manifold, and we have studied the dephasing of this quantum bit. In this case, due to the fact that successive atoms have different energies, each realization of the qubit evolves differently with respect to a local oscillator. This results in a loss of coherence, that we were nevertheless able to compensate for by applying rephasing "spin-echo" techniques. For this application, a lower energy spread of the trapped atom would also increase the dephasing time, thus avoiding the extra "spin-echo" sequence.

In the present paper, we analyze in more detail the methods used in these previous works to determine the mean energy of a single atom in the optical tweezer [15-17]. Similar or complementary techniques have been reported by other groups $[12,18]$. We then exploit these temperature measurements to characterize several cooling methods to further decrease the temperature of the atoms, and we show how to approach the ground state level in the two radial directions of the trap, which exhibit the strongest confinement.

The paper is organized as follows. We describe in Sec. II the experimental setup. In Sec. III, we present a release and recapture technique used to measure the temperature of the atoms. In Sec. IV we develop a cooling sequence used to laser-cool the atom in the tweezer. In Sec. V, we perform the spectroscopy of the energy distribution of the atom in the tweezer and find that this distribution is very close to a Boltzmann distribution. Section VI describes how the mean energy of the single atom can be decreased by truncating this Boltzmann distribution. This provides a nondeterministic method to prepare a single atom close to the ground state of the trapping potential. In Sec. VII we reduce further the temperature of the atoms by adiabatically lowering the potential trap. Finally, we conclude by discussing some implications of these results.

\section{EXPERIMENTAL SETUP}

Figure 1 shows a schematic of the experimental setup. A beam of ${ }^{87} \mathrm{Rb}$ atoms is slowed using a Zeeman slowing tech- 


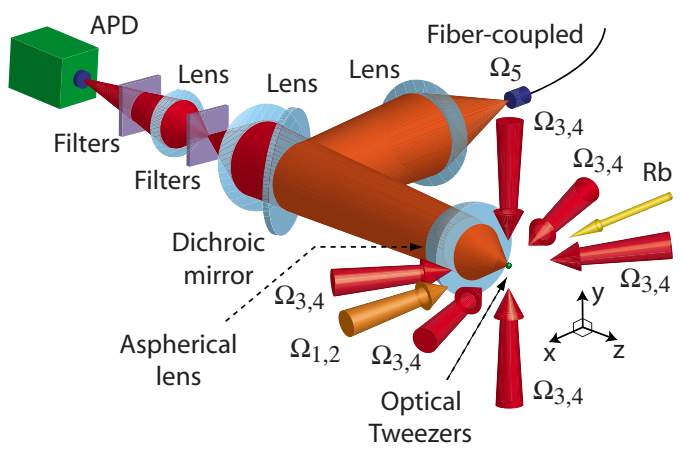

FIG. 1. (Color online) Schematic of the experimental setup. A beam of ${ }^{87} \mathrm{Rb}$ atoms, originating from an oven, is slowed using a Zeeman coil (not shown) and counter propagating slowing $\left(\Omega_{1}\right)$ and repumping $\left(\Omega_{2}\right)$ lasers at $\sim 780 \mathrm{~nm}$. An optical molasses is formed at the intersection of six counter propagating lasers $\left(\Omega_{3}\right)$ and repumpers $\left(\Omega_{4}\right)$ operating also at $\sim 780 \mathrm{~nm}$. An aspherical lens is used to tightly focus a $\sim 850 \mathrm{~nm}$ laser $\left(\Omega_{5}\right)$ in the region of the optical molasses. The fluorescence of the atom, due to the molasses and Zeeman lasers, is collected using the same aspherical lens. A dichroic mirror together with other spatial and spectral filters are used to filter the fluorescence signal, which is detected using singlephoton counting avalanche photodiode (APD).

nique [19]. The counterpropagating laser beam at $\sim 780 \mathrm{~nm}$ (denoted $\Omega_{1}$ ) is $18.7 \Gamma$ red detuned from the $F=2 \rightarrow F^{\prime}=3$ cycling transition, and combined with a repumping laser $\left(\Omega_{2}\right)$ that is $3.7 \Gamma$ red detuned from the $F=1 \rightarrow F^{\prime}=2$ repumping transition. Here, $\Gamma \approx 2 \pi \times 6 \times 10^{6} \mathrm{rad} / \mathrm{s}$ is the linewidth of the $D_{2}$ transition.

We form an optical molasses at the intersection of six counterpropagating cooling lasers that are $4.2 \Gamma$ red detuned from the $F=2 \rightarrow F^{\prime}=3$ transition (denoted $\Omega_{3}$ ), together with six repumping lasers $\left(\Omega_{4}\right)$ resonantly tuned to the $F=1$ $\rightarrow F^{\prime}=2$ transition. This optical molasses is used as a reservoir of cold atoms from which the dipole trap is loaded.

We produce the optical dipole trap by focusing a $\sim 850 \mathrm{~nm}$ laser in the center of the molasses, using a large numerical aperture $(\mathrm{NA}=0.5)$ aspherical lens [17]. The optical dipole trap has a measured optical waist of $w$ $=1.03 \pm 0.01 \mu \mathrm{m}$. A comprehensive characterization of the optical system and of the dipole trap itself is presented in detail elsewhere [17]. Assuming a Gaussian beam profile in the transverse direction for the dipole trap and a Lorentzian profile along the propagation axis, we calculate the radial and axial frequencies and find $\nu_{\perp} \sim 160 \mathrm{kHz}$ and $\nu_{\|}$ $\sim 30 \mathrm{kHz}$, respectively, for $10 \mathrm{~mW}$ of laser power. The potential trap depth, proportional to the laser power, is $U$ $\sim 2.8 \mathrm{mK}$ for a $10 \mathrm{~mW}$ of laser power.

This optical tweezer allows us to trap single ${ }^{87} \mathrm{Rb}$ atoms via a collisional blockade mechanism, which prevents two or more atoms from being trapped simultaneously due to inelastic collisions $[11,17,20]$. The $\sim 780 \mathrm{~nm}$ fluorescence of the trapped single atoms is detected using the same aspherical lens that is used to create the dipole trap. This fluorescence is filtered using a dichroic mirror, together with other spatial and spectral filters, and then detected using a single photon counter (avalanche photodiode), with an overall collection and detection efficiency that is estimated to be $\sim 0.5 \%$. From

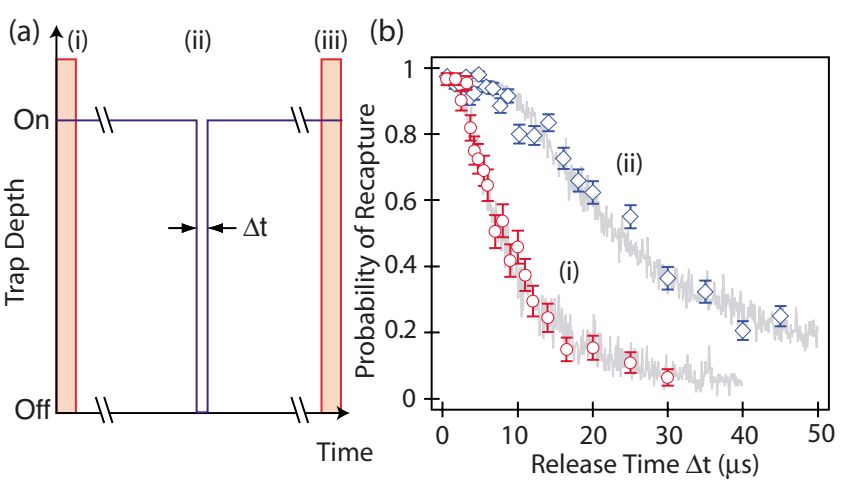

FIG. 2. (Color online) Measurement of the temperature of the atoms using the release and recapture method. (a) Schematic of the experimental sequence (see text). (b) Experimental results showing the probability of recapturing the atom $P_{\mathrm{RR}}(\Delta t)$ as a function of release time $\Delta t$. (i) (Circles) Temperature of the atoms just after the loading in the dipole trap. Each data point is the accumulation of 100 sequences. Superimposed on this data is a fit by the Monte Carlo simulation of the release and recapture method, which is the average of 200 trajectories for each release time. The temperature is $168 \pm 6 \mu \mathrm{K}$ for a trap depth of $\sim 2.8 \mathrm{mK}$. (ii) (Diamonds) For comparison we have also plotted the results for the temperature measurement using the same method, but after the atom has been laser cooled in the tweezer. Each data point is the accumulation of 200 sequences. The best-fit temperature is $31 \pm 1 \mu \mathrm{K}$ for a trap depth of $\sim 2.5 \mathrm{mK}$

the output signal of the single-photon counter we distinguish between the presence (or absence) of an atom in the tweezer, within a $10 \mathrm{~ms}$ acquisition period, with a confidence better than $99 \%$. Each experimental sequence that will be presented in this paper is triggered on a detected single atom in the dipole trap. Our goal in the following sections will be to investigate the energy distribution and temperature of the atoms trapped in the optical tweezer, under various cooling conditions.

\section{RELEASE AND RECAPTURE TECHNIQUE}

In this section we will describe a release and recapture method that we use to determine the temperature of the atoms in the dipole trap $[15,16]$. We emphasize that what we mean by the temperature of the atoms is a temperature extracted by averaging the energy over many realizations of the same experiment with a single atom. The premise of this method is that information can be obtained about the energy distribution of a single atom by switching off the dipole trap for a variable time $\Delta t$ [as illustrated in Fig. 2(a)] and then determining the probability of recapturing the atom, which is denoted as $P_{\mathrm{RR}}(\Delta t)$. On average, an atom with a high energy is more likely to escape during the release time $\Delta t$, compared to an atom with a low energy, as is well known in the context of laser cooling of atomic samples [21,22]. In the single atom regime used here, this method is particularly robust, since on each repetition of the experimental sequence the result is binary: either the atom has escaped the trapping region, or it is recaptured. This binarization of the measurement outcome makes it immune to various noise sources, in 
particular to fluctuations in the fluorescence level of the atom, which is used to decide on the presence of the atom.

Figure 2(a) illustrates a schematic of the release and recapture sequence, which goes as follows. (i) After the atom is trapped, the cooling and loading lasers $\left(\Omega_{1}, \Omega_{2}, \Omega_{3}\right.$, and $\left.\Omega_{4}\right)$ are switched off. (ii) The dipole trap laser $\left(\Omega_{5}\right)$ is then turned off for a time $\Delta t$. (iii) Finally, we perform a fluorescence detection by switching all the cooling and loading lasers back on, thus determining whether the atom has escaped or not. Figure 2 shows typical experimental results for the release and recapture technique, where the probability of recapturing the atom is plotted as a function of the release time $\Delta t$ (more details about this experiment will be presented in the following section).

In order to extract the temperature from these measurements, we perform Monte Carlo simulations of the trajectories of the single atoms, to determine the probability that the atoms are recaptured after a release time $\Delta t$. This simulation requires the knowledge of the energy distribution of the atom in the trap. We assume that we are in the harmonic approximation of the trapping potential, and that the positionvelocity distribution of the atom follows a thermal MaxwellBoltzmann law. The standard deviations of the position of the atom in the axial and radial directions of the trapping potential are $\Delta x_{\|}=\sqrt{k_{B} T / m \omega_{\|}^{2}}$ and $\Delta x_{\perp}=\sqrt{k_{B} T / m \omega_{\perp}^{2}}$, respectively, while the standard deviation of the velocity is $\Delta v$ $=\sqrt{k_{B} T / m}$, where $T$ is the temperature. The parameters of the optical trapping potential have been determined previously [17], and take into account the tilt due to gravity, which creates a local maximum in the potential along the gravity axis. The effective trap depth is defined as the height of this potential barrier with respect to the minimum of the potential.

The simulation goes as follows: using the MaxwellBoltzmann distribution we randomly generate the threedimensional position-velocity vector $\left(x_{i}, y_{i}, z_{i}, v_{x, i}, v_{y, i}, v_{z, i}\right)$ of the atom in the potential for a given temperature. We then calculate the trajectory of each atom during the release time $\Delta t$ in a single time-step calculation. The position of the atom after this release time is $\left(x_{i}+v_{x, i} \Delta t, y_{i}+v_{y, i} \Delta t-g \Delta t^{2} / 2, z_{i}\right.$ $\left.+v_{z, i} \Delta t\right)$, where the potential gradient due to gravity along the $y$ axis has been included. We finally determine the energy of each atom when the trap is turned back on. If the total energy of the atom after the time-of-flight is smaller than the effective trap depth, we consider that the atom is recaptured, otherwise it is considered to have escaped.

By simulating many trajectories of the single atoms for each release time $\Delta t$, we can numerically determine the probability of recapturing the atom as a function of this release time. These simulations are repeated for a range of different temperatures. We calculate the weighted least square value $\left(\chi^{2}\right)$ between the experimental data and the simulated results for each temperature. The chi-square value is defined as $\chi^{2}$ $=\Sigma_{i}\left\{\left[f_{i}-P_{\mathrm{RR}}\left(\Delta t_{i}\right)\right]^{2} / \sigma_{i}^{2}\right\}$, where $P_{\mathrm{RR}}(\Delta t)$ is the experimental data that has an uncertainty $\sigma$ and $f$ is the value predicted by the model. The best-fit temperature corresponds to the Boltzmann distribution that minimizes this least square value.

We performed the release and recapture technique to determine the temperature of the atoms just after they were loaded in the trap. Figure 2(b) shows the Monte Carlo simulation that best fits the corresponding experimental data. In this example, the best fit temperature is $T=168 \pm 6 \mu \mathrm{K}$ in a trap depth of $U \sim 2.8 \mathrm{mK}$. The ratio between the trap depth and the temperature of the atoms is 15 , indicating that we are approximately in the harmonic regime of the trapping potential.

Due to experimental miscounts and losses, the probability of recapturing an atom for zero release time was less than unity, being instead typically 0.95. We scaled the Monte Carlo simulation by this factor to achieve a better fit to the experimental results. The uncertainty on the temperature that minimized the chi-square value is defined as $\sigma_{T}$ $=\sqrt{2\left(\partial^{2} \chi^{2} / \partial T^{2}\right)^{-1}}$, assuming that the $\chi^{2}$ function varies harmonically around this minimum point [23]. Furthermore, we have included in the error budget for the temperature, the statistical uncertainty in fitting a parabola function to this chi-square minimum.

We will use this release and recapture technique throughout the rest of the paper as a diagnostic to measure the temperature of the atoms in the optical tweezer.

\section{RADIATIVE COOLING OF A SINGLE ATOM}

In this section, we discuss the laser cooling of a single atom after it has been captured in the dipole trap. For that purpose, the cooling sequence was optimized by controlling the molasses lasers parameters (cooling time, frequency detuning, intensity), as well as the trapping laser intensity. This was done by maximizing the recapture probability of the single atom after a release and recapture experiment with $\Delta t=10 \mu \mathrm{s}$.

Figure 3 shows the optimized laser cooling sequence. Initially, all lasers are on and the molasses cooling lasers are detuned by $\Delta_{1}=-4.5 \Gamma$ to maximize the loading rate of the molasses and optical dipole trap. (i) The sequence is triggered on the detection of an atom, where the dipole trap laser power is $\sim 10 \mathrm{~mW}$. (ii) The Zeeman laser and the Zeeman repumper laser are switched off. At the same time, the cooling lasers are linearly detuned from $\Delta_{1}=-4.5 \Gamma$ to $\Delta_{2}=-2 \Gamma$ in $\sim 1.2 \mathrm{~ms}$. During this time, the intensities of the molasses lasers are reduced from $I_{1} \sim 2 \mathrm{~mW} /$ beam, to approximately one third of their initial intensity. After this cooling period the cooling and repumping lasers are switched off.

Using this optimized laser-cooling sequence we were able to significantly reduce the temperature of the atoms. Figure 2(b) shows the release and recapture experimental results after the laser cooling, together with the best-fit simulation results. This corresponds to a temperature of $31 \pm 1 \mu \mathrm{K}$ for a trap depth of $\sim 2.5 \mathrm{mK}$, which yields the ratio $U / T \sim 81$. This represents more than a factor 5 reduction in the temperature with respect to the temperature of the atoms directly loaded from the optical molasses.

We note that the optimized cooling sequence is achieved when the laser detuning is brought closer to resonance with respect to the atomic transition. This fact may seem inconsistent with the sub-Doppler theory of laser cooling (see, e.g., Ref. [24]), which predicts that the temperature is lower for larger detuning. However, one has to take into account 


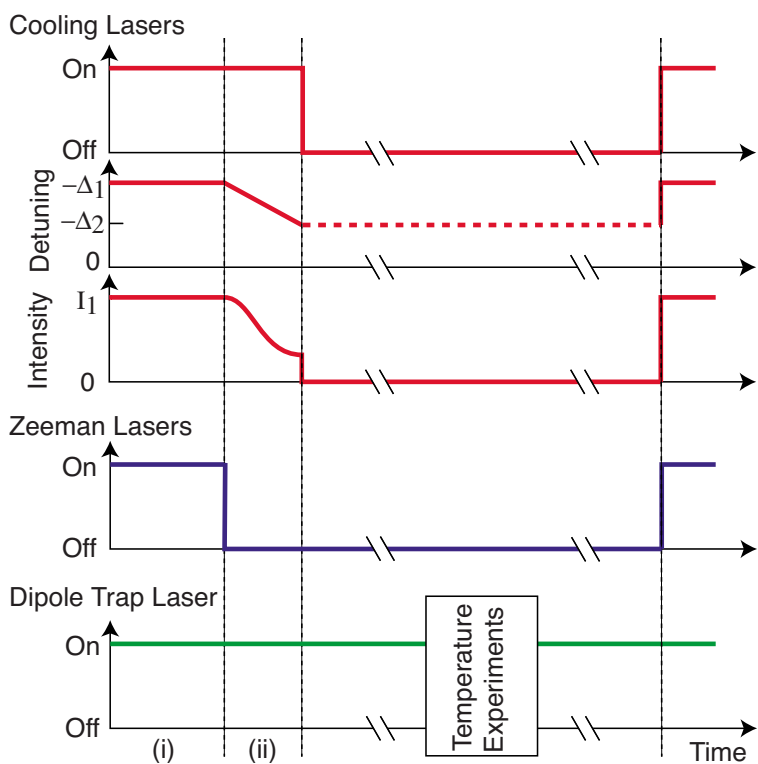

FIG. 3. (Color online) Schematic of the temporal sequence for the laser cooling sequence. The details are given in the body of the text. The box labeled "temperature experiments" shows the point in the sequence where we can manipulate the intensity of the dipole trap laser in order to measure the temperature of the atoms (see Secs. V-VII).

that when the atom is trapped in the tweezer, the resonance frequency is light shifted by about $10 \Gamma$ with respect to the free space case. Therefore the detuning experienced by the trapped atom varies only from $-14.5 \Gamma$ to $-12 \Gamma$. The extra cooling effect is then attributed to the decrease (by a factor 3 ) in the intensity of the laser beams, rather than to the frequency change, now in reasonable agreement with subDoppler laser cooling.

This optimized cooling technique is the starting point for each experiment presented throughout the rest of the paper. We will now turn our attention to validating the assumption about the thermal distribution. In addition, we would like to cross-check the temperature results using an alternative method.

\section{ENERGY DISTRIBUTION OF A SINGLE ATOM IN AN OPTICAL TWEEZER}

In this section we determine the energy distribution of the single atom in the dipole trap. Provided that this distribution is indeed thermal, the corresponding temperature can be evaluated. To achieve this, we apply a type of spectroscopy method proposed and experimentally demonstrated by Alt et al. in Ref. [18].

This method involves the adiabatic lowering of the trap depth to a point where the atom can potentially escape. As shown in Fig. 4(a), by lowering the trap depth, an atom with initial energy $E_{i}$ in a trap of depth $U_{i}$ will eventually have an energy $E_{\text {esc }}$ that is equal to the final trap depth $U_{\text {esc }}$ and hence the atom will escape the trap. Thus, measuring the depth at which the atom escapes the trap yields information about the initial energy of the atom.
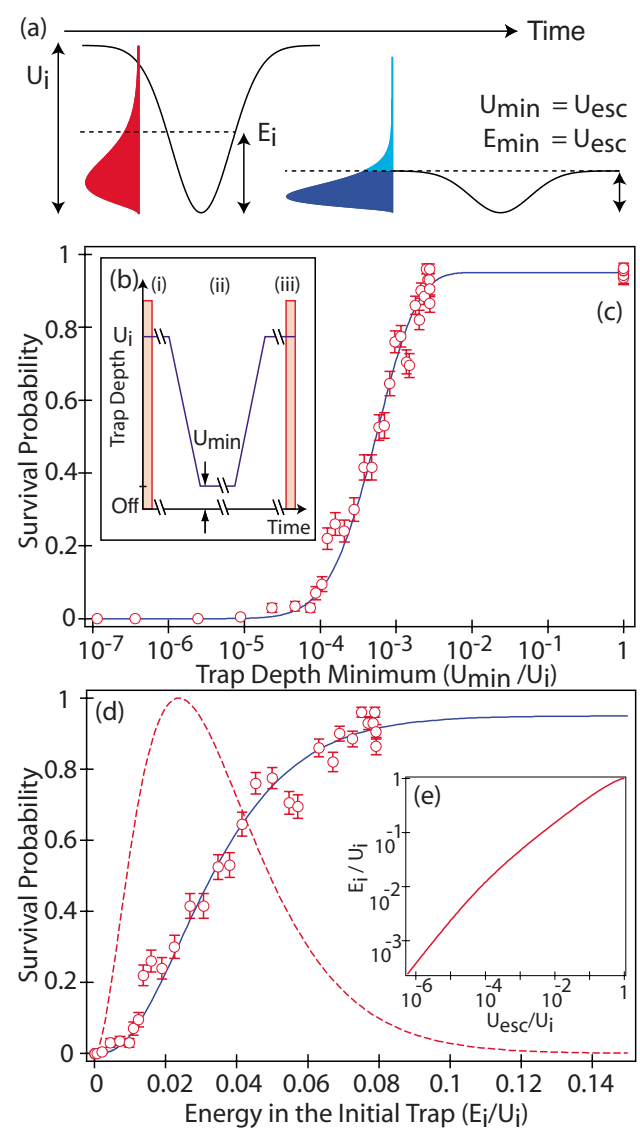

FIG. 4. (Color online) Spectroscopy of the energy distribution of a single atom using the adiabatic lowering method. (a) Schematic showing that due to the adiabatic lowering of the dipole trap, an atom with initial energy $E_{i}$ in a trap depth $U_{i}$ eventually has an energy equal to the trap depth, i.e., $E_{\min }=U_{\min }=U_{\text {esc. }}$ (b) Schematic of the experimental sequence (see text). (c) Experimental results of the survival probability for various minimum trap depths $U_{\text {min }}$. Each data point is the accumulation of 200 sequences, with error bars due to the binomial statistics. Solid line is a theoretical fit to the data. (d) Reconstruction of the Boltzmann distribution in the initial trap of depth $U_{i}$. Plot showing the percentage of atoms with an energy less than $E_{i}$ (in the initial trap of depth $U_{i}$ ) versus the normalized energy $E_{i} / U_{i}$. Solid line is a theoretical fit to the data (see text). Dashed line represents the corresponding Boltzmann distribution (e) Numerical results for solving the constant action equation $S\left(E_{i}, U_{i}\right)=S\left(U_{\mathrm{esc}}, U_{\mathrm{esc}}\right)$.

Figure 4(b) shows a schematic of the experimental sequence for this method, which goes as follows: (i) The single atom is trapped and laser cooled. (ii) The dipole trap depth is then adiabatically lowered from $U_{i} \sim 2.8 \mathrm{mK}$ to $U_{\min }$. The lowering time is approximately $\sim 2.5 \mathrm{~ms}$. The trap depth is held constant for a duration of $\sim 20 \mathrm{~ms}$, to allow an atom with an energy greater than the trap depth sufficient time to escape [30]. (iii) After this waiting time, the trap depth is adiabatically raised to the initial trap depth $U_{i}$ in $\sim 2.5 \mathrm{~ms}$. Finally, the laser beams are switched back on to determine if the atom has escaped or not. We leave to Sec. VII the discussion about the adiabaticity of this process. Here we assume that the hypothesis for the adiabatic change of the potential is fulfilled. Figure 4(c) shows the experimental 
probability of recapture for various minimum trap depths $U_{\text {min }}$ normalized to the initial trap depth $U_{i}$ [31].

It is now necessary to map the minimum trap depth $U_{\text {min }}$ back to the corresponding energy in the initial trap $E_{i}$. To perform this mapping we calculate the one-dimensional action that can be expressed as the integral $S(E, U)$ $=\int_{0}^{x_{\max }} \sqrt{2 m[E-U(x)]} d x$, where $E$ is the energy, $U(x)$ is the potential and $x_{\max }$ is the position where the atom has zero kinetic energy. In the adiabatic limit, the action of the atom in the trap is conserved as the trap depth is adiabatically lowered [25]. We solve the constant action equation $S\left(E_{i}, U_{i}\right)=S\left(U_{\text {esc }}, U_{\text {esc }}\right)$ in the radial direction of our potential trap that includes the gravity potential gradient. The numerical results of the $U_{\mathrm{esc}} / U_{i} \rightarrow E_{i} / U_{i}$ mapping is plotted in Fig. 4(e). We subsequently apply the mapping $U_{\mathrm{esc}} / U_{i} \rightarrow E_{i} / U_{i}$ to our experimental data. Figure 4(d) shows the percentage of atoms that have an energy less than $E_{i}$ in the initial trap of depth $U_{i}$ plotted as a function of the normalized energy $E_{i} / U_{i}$.

We now wish to compare the measured energy distribution to a thermal one. In the harmonic trap limit (i.e., when the ratio of the trap depth to the temperature of the atoms satisfies $U_{i} / k_{B} T \gg 1$ ), we calculate the Boltzmann energy distribution for a temperature $T$. We assume that the normalized energy distribution of the atom is

$$
f_{\text {th }}(E)=\frac{1}{2\left(k_{B} T\right)^{3}} E^{2} e^{-E / k_{B} T},
$$

where $E^{2}$ corresponds to the three-dimensional density of states. The probability that an atom has an energy less than $E$ is defined by $P_{\text {surv }}(E)=\int_{0}^{E} f_{\text {th }}\left(E^{\prime}\right) d E^{\prime}$ and is given by

$$
P_{\text {surv }}(E)=1-\left[1+\eta+\frac{1}{2} \eta^{2}\right] e^{-\eta},
$$

where $\eta=E / k_{B} T$. Equation (2) can be interpreted as the survival probability of the atom remaining in the dipole trap after the truncation of the Boltzmann distribution.

Figure 4(d) shows a fit of the integrated Boltzmann distribution $P\left(E_{i} / U_{i}\right)$ to the experimental data using Eq. (2). To achieve a better fit to the data, the maximum survival probability is rescaled to 0.95 , to account for nonideal recapture probability of our experiment when $U_{\min }=U_{i}$. We find that the measured energy distribution is well fitted by a thermal distribution. This fact shows that over many realizations of the experiments, the energy of successive atoms after laser cooling is well described by a Boltzmann distribution, in agreement with several previous theoretical and experimental studies [26].

The Boltzmann distribution in Fig. 4(d) has a corresponding temperature of $T=33 \pm 2 \mu \mathrm{K}$ for a trap depth $U_{i}$ $\sim 2.8 \mathrm{mK}$, where the error in the temperature corresponds the statistical uncertainty in the fit. This temperature is in good agreement with the release and recapture temperature results presented in the previous section.

We point out that the adiabatic lowering of the dipole trap depth is a powerful tool in manipulating the energy distribution of the atom. For example, at very low trap depths the more energetic atoms will escape with a higher probability.
This adiabatic lowering indeed acts as a filter such that only the coolest atoms will remain in the trap, as we will now show.

\section{REDUCTION OF MEAN ENERGY BY TRUNCATING THE BOLTZMANN DISTRIBUTION}

We have shown in Secs. IV and V that we can cool the atoms down to a temperature of approximately $T=33 \mu \mathrm{K}$ using laser cooling techniques. It is possible to further reduce the mean energy of the trapped atom by truncating the Boltzmann distribution at an energy $U_{\text {trunc }}$. Here, we perform this truncation by adiabatically lowering the trapping potential, to a point where atoms with a higher energy are likely to escape, while atoms with a lower energy remain trapped. This filtering via the lowering of the trap potential appears to be similar to evaporative cooling, but a crucial difference is that there is no collision-induced rethermalization, since the experiment is done at the single atom level [27].

Figure 5(a) shows a schematic of the experimental sequence that we use to investigate the energy distribution of the truncated Boltzmann distribution. This experimental sequence is identical to the one described in Sec. V, but we now perform a release and recapture sequence directly after it. The entire temporal sequence is shown in the inset of Fig. 5(b). The additional release and recapture sequence allows us to determine the temperature of the atoms after the truncation process and after the trap depth has been returned to the initial depth.

To determine the truncation energy $U_{\text {trunc }}$ in the trap of potential depth $U_{f}=U_{i}$, we first calculate the truncation energy in the shallow trap of potential depth $U_{\text {min }}$ (using the measured minimum optical dipole trap power). We then use the numerical results that map $U_{\min } \rightarrow E_{i}$ [see Fig. 4(e)] to determine the truncation energy $U_{\text {trunc }}$ in the trap of potential depth $U_{f}$

In the case of a truncated Boltzmann distribution, it is not sufficient to assume that the position-velocity vector of the atom follows a Gaussian Maxwell Boltzmann distribution, as we did for the release and recapture experiments in Secs. III and IV. To account for this effect, in the following simulations we consider that the energies of the atoms are drawn from a truncated Boltzmann distribution corresponding to a temperature $T$ and a truncation energy $U_{\text {trunc. The Boltzmann }}$ distribution is discretized into $N$ bins with energies $E_{j}$ equally spaced by $\Delta U=U_{\text {trunc }} / N$, for $\Delta U / 2 \leqslant E_{j} \leqslant\left(U_{\text {trunc }}\right.$ $-\Delta U / 2)$. For each distribution, the number of simulations with atoms with an energy $E_{j}$ is weighted according to this discretized Boltzmann distribution. For an atom with energy $E_{j}$, we randomly distribute the energies among the three directions given by the Cartesian axes. The period of motion of the atom is then randomly chosen for each of the three Cartesian axes (ensuring that the total energy of the atom remains $\left.E_{j}\right)$. Finally, the trajectories of the atoms are simulated during the time of flight as described in Sec. III. We typically use several hundred atom trajectories for each release time $\Delta t$, and we discretize the Boltzmann distribution into 10 bins. When the truncation energy is very large with respect to the temperature (i.e., the distribution is approximately not 

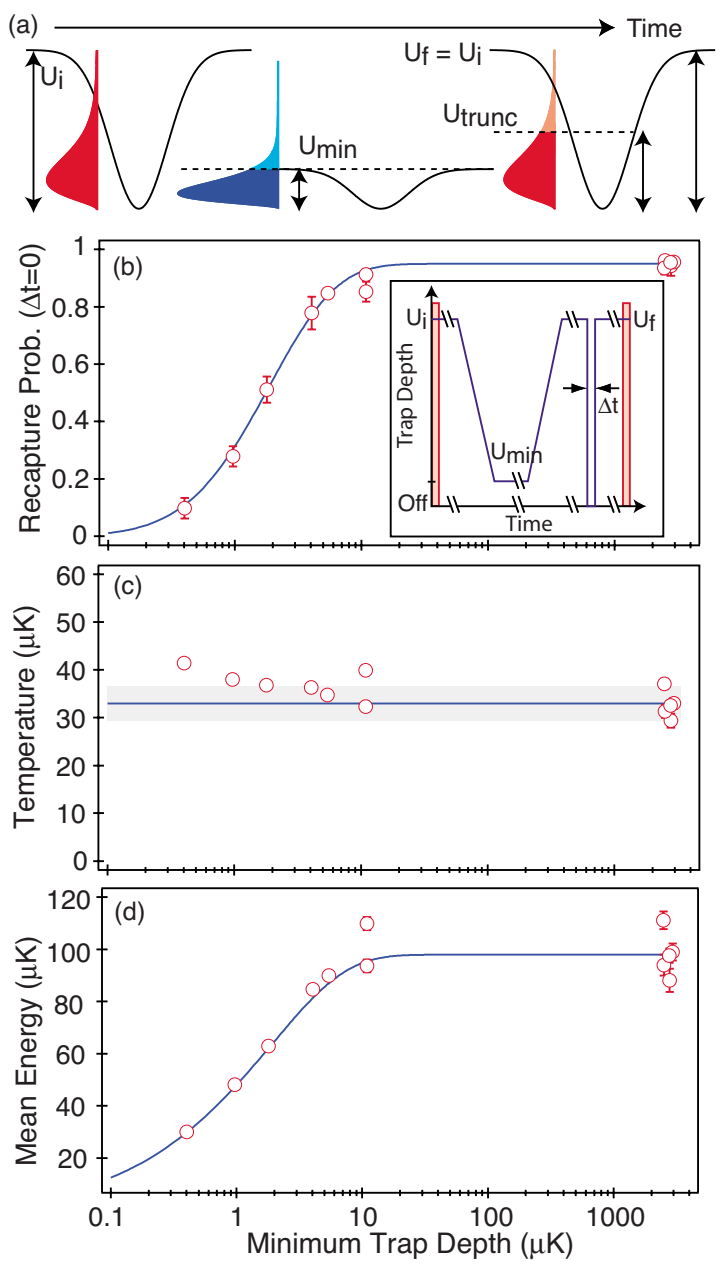

FIG. 5. (Color online) Investigation of a truncated Boltzmann distribution using the release and recapture method. (a) Schematic of the experimental sequence. (b) Probability of recapture for zero release time $P_{\mathrm{RR}}(\Delta t=0)$ plotted as a function of the minimum trap depth $U_{\text {min }} . P_{\mathrm{RR}}(\Delta t=0)$ is defined as the mean of the first four recapture probabilities with the smallest release times of a given data set [e.g., see, Fig. 2(b)], where the error bars are due to normal statistics. Solid line is a theoretical fit to the data. The inset shows a schematic of the temporal experiment sequence. (c) The temperature $T$ plotted as a function of $U_{\min }$. Solid line corresponds to a constant temperature of $33 \mu \mathrm{K}$. Gray bar corresponds to the standard deviation of the spread in the measured temperatures for the minimum trap depth $U_{\min } \sim 2.8 \mathrm{mK}$. (d) The calculated mean energy $\langle E\rangle$ of the truncated Boltzmann distribution shown as a function of $U_{\text {min }}$. The solid line is a theoretical prediction of the mean energy of a truncated Boltzmann distribution with a temperature of $33 \mu \mathrm{K}$.

truncated), this new model yields the same results as the previous release and recapture model.

The experimental results are shown in Fig. 5. We repeated the experiment several times for different minimum trap depths $U_{\text {min }}$. The data were recorded over a span of several days, where all other parameters were kept approximately constant. For each minimum trap depth we measured the recapture probability, $P_{\mathrm{RR}}(\Delta t=0)$, the temperature $T$ of the truncated Boltzmann distribution and we calculated the associated mean energy $\langle E\rangle$ of this distribution. The small day to day variations in the experimental parameters result in a small spread of the temperatures for a given value of $U_{\min }$. Nevertheless, Fig. 5(c) shows that the temperature of the Boltzmann distribution remains approximately constant down to very small trap depths, even when the distribution becomes truncated.

In contrast, the mean energy is reduced markedly as the distribution becomes more truncated, as we will now describe. The mean energy of a truncated Boltzmann distribution in a three-dimensional harmonic potential can be expressed as

$$
\begin{aligned}
\langle E\rangle= & \frac{\int_{0}^{U_{\text {trunc }}} E^{3} e^{-E / k_{B} T} d E}{\int_{0}^{U_{\text {trunc }}} E^{2} e^{-E / k_{B} T} d E} \\
& =3 k_{B} T\left[\frac{1-\left(1+\eta+\frac{1}{2} \eta^{2}+\frac{1}{6} \eta^{3}\right) e^{-\eta}}{1-\left(1+\eta+\frac{1}{2} \eta^{2}\right) e^{-\eta}}\right],
\end{aligned}
$$

where $\eta=U_{\text {trunc }} / k_{B} T$. In the limit where the Boltzmann distribution is approximately nontruncated, i.e., $\eta \gg 1$, the mean energy is given by $\langle E\rangle=3 k_{B} T$. However, as shown in Eq. (3) the mean energy is reduced significantly as the truncation is increased, i.e., $\eta \rightarrow 0$. The probability that the atom survives this truncation process is given by Eq. (2) as $P_{\text {surv }}\left(U_{\text {trunc }}\right)$. Naturally, this probability drops quickly as $\eta \rightarrow 0$, thus reducing the efficiency (or effective duty cycle) of this scheme.

Figure 5(d) shows the mean energy calculated using Eq. (3) with the measured values of the temperature and the truncation energy $U_{\text {trunc }}$. The solid line is a theoretical prediction using Eq. (3) for a temperature $T=33 \mu \mathrm{K}$.

For minimum trap depths above $10 \mu \mathrm{K}$, i.e., when the energy distribution is approximately nontruncated, the mean energy is $\langle E\rangle \sim 100 \mu \mathrm{K}$. For minimum trap depths below $10 \mu \mathrm{K}$, we see that the mean energy is significantly reduced. For example, for a minimum trap depth $U_{\min } \sim 0.4 \mu \mathrm{K}$, we measure a mean energy of the atom at the end of this filtering sequence of $\langle E\rangle \sim 30 \mu \mathrm{K}$, where the final trap depth is $U_{f}$ $\sim 2.8 \mathrm{mK}$. The truncation of the Boltzmann distribution thus leads to a reduction of the mean energy by a factor of $100 \mu \mathrm{K} / 30 \mu \mathrm{K} \sim 3.3$. At the same time, the probability of recapture is reduced to $P_{\mathrm{RR}}(\Delta t=0) \sim 0.1$.

In this section we have shown that if one is willing to compromise on the probability of recapturing the atom (or, correspondingly, the duty cycle of the experiment), truncating the Boltzmann distribution is a good way to reduce the mean energy of the atom.

\section{COOLING A SINGLE ATOM BY ADIABATICALLY LOWERING THE TRAPPING POTENTIAL}

Let us consider an atom in a trapping potential. By adiabatically lowering the trap depth the occupation probabilities of the vibrational levels are preserved [28]. For our optical dipole trap this conservation of the vibrational number re- 
(a)
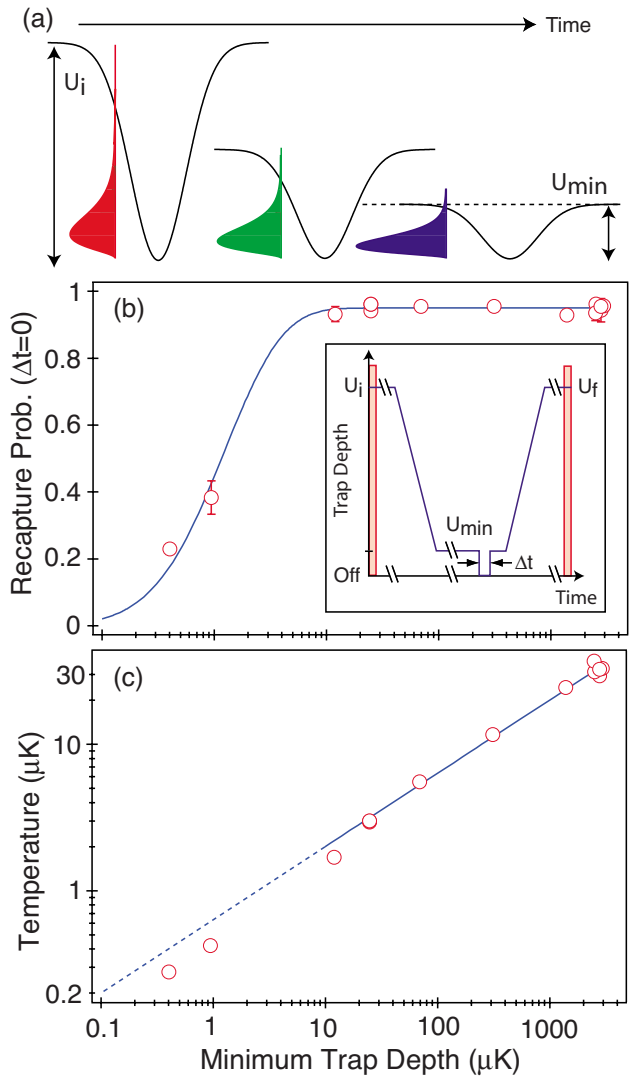

FIG. 6. (Color online) Investigation of the adiabaticity during the change of the optical trap depth. (a) Schematic of the experimental sequence (see text). (b) Probability of recapture for zero release time $P_{\mathrm{RR}}(\Delta t=0)$, as a function of the minimum trap depth $U_{\text {min }}$. The solid line is a theoretical fit to the data. Inset shows a schematic of the temporal experimental sequence. (c) Temperature $T$ of the Boltzmann distribution plotted as a function of $U_{\text {min }}$. The solid line is a theoretical prediction of the adiabatic scaling law $T / \sqrt{U}=$ const. The dashed line is the extension of the solid line to minimum trap depths at which the density of states deviates from that of a three-dimensional harmonic trap.

sults in $T / \sqrt{U}$ being a constant. In this section we will experimentally demonstrate that by slowly lowering the trap depth, the temperature of the atoms follows this relation, thus reinforcing our assumptions about adiabaticity.

To test the adiabaticity of our lowering scheme we perform the same experimental sequence as in Sec. VI, but we now implement the release and recapture sequence when the trap is shallowest, i.e., we measure the temperature of the atoms at the minimum trap depth $U_{\min }$ [32]. This is in contrast to Sec. VI, where the temperature of the atoms was measured after the trap depth was returned to its initial value.

A schematic of the experimental sequence is shown in Fig. 6, together with the recapture probability $P_{\mathrm{RR}}(\Delta t=0)$ and the temperature $T$ measured for various minimum trap depths $U_{\text {min }}$. The data in Fig. 6(c), shows evidence that the temperature of the Boltzmann distribution follows closely an adiabatic lowering behavior. For comparison, we have plotted the adiabatic scaling law $T / \sqrt{U}=$ const, where the constant is equal to $T_{i} / \sqrt{U_{i}}$ with $T_{i}=33 \mu \mathrm{K}$ and $U_{i}=2.8 \mathrm{mK}$.

The criteria for adiabaticity states that the rate of change of the oscillation frequency $\dot{\omega}$ must be lower than $\omega^{2}(\omega$ is the oscillation frequency) at all times during the evolution [25]. Care must be taken to fulfill the adiabaticity criteria when the trapping potential is very small, corresponding to a small oscillation frequency. The rate of change of the oscillation frequency must be sufficiently small in this case. In our experiment, this is done by smoothly ramping up and down the intensity of the dipole trap laser with acousto-optic modulators. The behavior of the measured temperature in Fig. 6(c) gives us a strong indication that indeed the adiabaticity criteria is fulfilled when the trapping depth is small.

Figure 6(b) shows the probability of recapture for zero release time $P_{\mathrm{RR}}(\Delta t=0)$ plotted as a function of the minimum trap depth $U_{\text {min }}$, together with a theoretical fit. We reemphasize that for minimum trap depths less than $U_{\text {min }}$ $\sim 10 \mu \mathrm{K}$, the Boltzmann distribution becomes truncated. This implies that we are no longer in the three-dimensional harmonic approximation, and hence the density of states is no longer described by $\rho(E) \propto E^{2}$. Nevertheless, for clarity in Fig. 6, we have presented the results assuming that $\rho(E)$ $\propto E^{2}$ for all data points.

As a typical result, we measure a temperature of $T$ $=1.69 \pm 0.02 \mu \mathrm{K}$ for the atoms in a trap that has been adiabatically lowered to a depth of $U_{\min } \sim 12 \mu \mathrm{K}$. In this case, the Boltzmann distribution is only slightly truncated, implying that the quadratic density of states should still be valid. This temperature corresponds to a one hundred fold decrease in the temperature of the atoms compared to those that were directly loaded from the molasses.

\section{DISCUSSION}

We have shown that the single atom cooling via filtering techniques is quite efficient. Starting from an initial mean energy of $100 \mu \mathrm{K}$ after the laser cooling phase, we get a final mean energy of the atom of $30 \mu \mathrm{K}$ with a probability of $\sim 0.1$. On our experiment, the loading rate of single atoms is on the order of 1 per second. If we are willing to comprise our duty cycle, we could obtain on average one atom every 10 seconds with a mean energy of $30 \mu \mathrm{K}$ in a $\sim 2.8 \mathrm{mK}$ deep trap, which corresponds to a ratio of the trap depth to mean energy of $\sim 93$.

Another parameter characterizing the external motion of the atom in the trap is the mean vibrational number along one axis, defined as the ratio of the mean energy of the atom along this axis to the energy difference between vibrational states. As the trap has two different oscillation frequencies along the axial and radial directions, this corresponds to two different mean vibrational numbers along these directions. If we assume the equipartition of the mean energy among the three axis, we get for the mean vibrational number along the radial direction $n_{\perp}=\langle E\rangle / 3 h \nu_{\perp}$. For the mean energy of $100 \mu \mathrm{K}$ that we obtained after laser cooling the atom, this leads to $n_{\perp} \sim 4.3$. By contrast, the lowest mean energy of $30 \mu \mathrm{K}$ that we obtained after filtering leads to $n_{\perp} \sim 1.3$, which is 3.3 times smaller than the mean vibrational number before filtering. This mean number, close to 1 along the radial direction, raises some questions about the validity of our classical treatment for the temperature measurement for the lowest trap depths. This analysis, albeit interesting, is beyond 
the scope of this paper. This also places us in a good position to further cool the atom down to the ground state, for example by using Raman sideband cooling [5].

In the framework of quantum information, there has been proposed a protocol to entangle two atoms based on the emission of a single photon by one of the two trapped atoms [29]. A crucial feature of this proposal is the ability to localize each atom within a distance smaller than the wavelength of the emitted photon, the so-called Lamb-Dicke regime. Reference [29] shows that for atoms described by a thermal distribution at temperature $T$, this condition becomes $\eta_{\text {th }}$ $=\eta_{\mathrm{LD}} \sqrt{k_{B} T / \hbar \omega} \ll 1$, with $\omega$ being the mean oscillation frequency of the atom in the trap, and $\eta_{\mathrm{LD}}=\sqrt{E_{r} / \hbar \omega}$ being the Lamb-Dicke parameter ( $E_{r}$ is the recoil energy). In our case, for a trap depth of $\sim 2.8 \mathrm{mK}$, we find $\eta_{\mathrm{LD}}=0.20$ and $\eta_{\mathrm{th}}$ $=0.56$ for a temperature of $33 \mu \mathrm{K}$, as measured above. We are therefore in the right regime to apply this entanglement protocol. We finally note that $\eta_{\text {th }}$ can be lowered when increasing the trap depth adiabatically. In this case $\eta_{\text {th }}$ is related to the trap depth $U$ by $\eta_{\text {th }} \propto U^{-1 / 4}$.

\section{CONCLUSION}

As a summary of this paper, we have investigated the energy of a single atom trapped in a tight optical dipole trap.
We have found that after applying laser cooling of the atom in the tweezer, the energy of the trapped atom follows a Boltzmann distribution. We have extracted the temperature of the atoms from the measured energy distribution. We have also described a release and recapture method to measure the temperature of the atoms assuming a truncated Boltzmann distribution. This second method is in good agreement with the first one.

We have shown that by adiabatically lowering the trap depth we have reduced the temperature of the atoms down to several micro-K. Finally, we have cooled the atom by filtering the hottest atoms over many realizations of the same experiment, the equivalent of evaporative cooling but at the single atom level. This leads to an atom close to the ground state in the radial direction of the trap.

\section{ACKNOWLEDGMENTS}

We acknowledge support from the Integrated Project "SCALA" which is part of the European IST/FET/QIPC Program, from Institut Francilien des Atomes Froids (IFRAF), from ARDA/DTO, and from PPF-ENS. Y. R. P. Sortais was supported by IFRAF and CNRS, A. M. Lance also by IFRAF and by the Marie Curie program of the European Commission.
[1] H. Dehmelt, Rev. Mod. Phys. 62, 525 (1990).

[2] W. Neuhauser, M. Hohenstatt, P. E. Toschek, and H. Dehmelt, Phys. Rev. A 22, 1137 (1980).

[3] W. Nagourney, J. Sandberg, and H. Dehmelt, Phys. Rev. Lett. 56, 2797 (1986).

[4] J. C. Bergquist, R. G. Hulet, W. M. Itano, and D. J. Wineland, Phys. Rev. Lett. 57, 1699 (1986).

[5] F. Diedrich, J. C. Bergquist, W. M. Itano, and D. J. Wineland, Phys. Rev. Lett. 62, 403 (1989).

[6] M. Nielsen and I. Chuang, Quantum Computation and Quantum Information (Cambridge University Press, Cambridge, UK, 2000).

[7] J. Benhelm, G. Kirchmair, C. F. Roos, and R. Blatt, Nat. Phys. (to be published).

[8] Z. Hu and H. J. Kimble, Opt. Lett. 19, 1888 (1994).

[9] F. Ruschewitz, D. Bettermann, J. L. Peng, and W. Ertmer, Europhys. Lett. 34, 651 (1996).

[10] D. Frese, B. Ueberholz, S. Kuhr, W. Alt, D. Schrader, V. Gomer, and D. Meschede, Phys. Rev. Lett. 85, 3777 (2000).

[11] N. Schlosser, G. Reymond, I. Protsenko, and P. Grangier, Nature (London) 411, 1024 (2001).

[12] M. Weber, J. Volz, K. Saucke, C. Kurtsiefer, and H. Weinfurter, Phys. Rev. A 73, 043406 (2006).

[13] D. D. Yavuz, P. B. Kulatunga, E. Urban, T. A. Johnson, N. Proite, T. Henage, T. G. Walker, and M. Saffman, Phys. Rev. Lett. 96, 063001 (2006).

[14] O. Mandel, M. Greiner, A. Widera, T. Rom, T. W. Hänsch, and I. Bloch, Nature (London) 425, 937 (2003).

[15] J. Beugnon, M. P. A. Jones, J. Dingjan, B. Darquié, G. Messin, A. Browaeys, and P. Grangier, Nature (London) 440, 779
(2006)

[16] M. P. A. Jones, J. Beugnon, A. Gaëtan, J. Zhang, G. Messin, A. Browaeys, and P. Grangier, Phys. Rev. A 75, 040301(R) (2007).

[17] Y. R. P. Sortais, H. Marion, C. Tuchendler, A. M. Lance, M. Lamare, P. Fournet, C. Armellin, R. Mercier, G. Messin, A. Browaeys, and P. Grangier, Phys. Rev. A 75, 013406 (2007).

[18] W. Alt, D. Schrader, S. Kuhr, M. Müller, V. Gomer, and D. Meschede, Phys. Rev. A 67, 033403 (2003).

[19] W. D. Phillips and H. Metcalf, Phys. Rev. Lett. 48, 596 (1982).

[20] N. Schlosser, G. Reymond, and P. Grangier, Phys. Rev. Lett. 89, 023005 (2002).

[21] S. Chu, L. Hollberg, J. E. Bjorkholm, A. Cable, and A. Ashkin, Phys. Rev. Lett. 55, 48 (1985).

[22] P. D. Lett, R. N. Watts, C. I. Westbrook, W. D. Phillips, P. L. Gould, and H. J. Metcalf, Phys. Rev. Lett. 61, 169 (1988).

[23] P. R. Bevington and D. K. Robinson, Data Reduction and Error Analysis for the Physical Sciences (McGraw-Hill, New York, 2003).

[24] M. Drewsen, Ph. Laurent, A. Nadir, G. Santarelli, A. Clairon, Y. Castin, D. Grison, and C. Salomon, Appl. Phys. B: Lasers Opt. 59, 283 (1994).

[25] L. D. Landau and E. M. Lifschitz, Mechanics (Pergamon, New York, 1976).

[26] P. D. Lett, W. D. Phillips, S. L. Rolston, C. E. Tanner, R. N. Watts, and C. I. Westbrook, J. Opt. Soc. Am. B 6, 2084 (1989).

[27] O. J. Luiten, M. W. Reynolds, and J. T. M. Walraven, Phys. Rev. A 53, 381 (1996).

[28] F. Reif, Fundamentals of Statistical and Thermal Physics 
(McGraw-Hill, New York, 1965)

[29] C. Cabrillo, J. I. Cirac, P. Garcia-Fernandez, and P. Zoller, Phys. Rev. A 59, 1025 (1999).

[30] We assume that an atom with an energy greater than the trap depth has sufficient time to exit the trap provided the following condition is fulfilled: $1 / \tau \ll \nu_{\|}$, where $\tau$ is the time the atom is in the trap and $\nu_{\|}$is the trap frequency in the axial direction. This condition is satisfied down to a final trap depth $U_{f} / U_{i}$ $\sim 10^{-4}$, where $\tau \times \nu_{\|} \sim 10 \gg 1$.

[31] For the effective minimum trap depth $U_{\min }$ taking gravity into account turns out to be important for trap depths less than $\sim 10 \mu \mathrm{K}$ because the gravity potential acts to tilt the trapping potential, thus lowering the potential barrier along the gravity axis.

[32] After the release and recapture sequence we needed to bring the trap depth close to its initial value to measure if the atom is still present in the trap, otherwise the lasers sent on the atom would kick it out of the shallow trap too quickly to measure its presence. 